This is an electronic reprint of the original article. This reprint may differ from the original in pagination and typographic detail.

Author(s): Airaksinen, Tuomas; Heikkola, Erkki; Toivanen, Jari

Title: Local control of sound in stochastic domains based on finite element models

Year: $\quad 2011$

Version:

Please cite the original version:

Airaksinen, T., Heikkola, E., \& Toivanen, J. (2011). Local control of sound in stochastic domains based on finite element models. Journal Of Computational Acoustics, 19(2), 205-217. https://doi.org/10.1142/S0218396X11004432

All material supplied via JYX is protected by copyright and other intellectual property rights, and duplication or sale of all or part of any of the repository collections is not permitted, except that material may be duplicated by you for your research use or educational purposes in electronic or print form. You must obtain permission for any other use. Electronic or print copies may not be offered, whether for sale or otherwise to anyone who is not an authorised user. 


\title{
Local control of sound in stochastic domains based on finite element models*
}

\section{TUOMAS AIRAKSINEN† ERKKI HEIKKOLA JARI TOIVANEN ${ }^{\S}$}

June 27, 2011

\begin{abstract}
A numerical method for optimizing the local control of sound in a stochastic domain is developed. A three-dimensional enclosed acoustic space, for example, a cabin with acoustic actuators in given locations is modeled using the finite element method in the frequency domain. The optimal local noise control signals minimizing the least square of the pressure field in the silent region are given by the solution of a quadratic optimization problem. The developed method computes a robust local noise control in the presence of randomly varying parameters such as variations in the acoustic space. Numerical examples consider the noise experienced by a vehicle driver with a varying posture. In a model problem, a significant noise reduction is demonstrated at lower frequencies.
\end{abstract}

Keywords: local sound control; Stochastic domain; Helmholtz equation; finite element method; passenger car; quadratic optimization

\section{Introduction}

Machine generated noise is an increasing problem in modern working environments. Rotating and constantly moving parts such as wheels, engines and cooler fans are typical noise sources. Noise control applications are found especially in factory environment, engineering vehicles and passenger cars. It is possible to reduce noise significantly by different methods, which are often classified as either active or passive techniques $[4,13]$. Probably the best situation would be to remove or reduce important noise source mechanisms by suitable design choices such that noise control measures would become unnecessary. In many cases,

*Post-print of the article published in Journal of Computational Acoustics, Vol. 19, No. 2 (2011) 205-217. DOI: $10.1142 /$ S0218396X11004432

†Department of Mathematical Information Technology, University of Jyväskylä, P.O. Box 35 (Agora), , FI-40014 University of Jyväskylä, Finland, tuomas.a.airaksinen@jyu.fi

$\ddagger$ Numerola Oy, P.O. Box 126, FI-40101 Jyväskylä, Finland, erkki.heikkola@numerola.fi

$\S$ Institute for Computational and Mathematical Engineering, Durand Building, Stanford University, Stanford, CA 94305, USA, toivanen@stanford.edu 
however, this is not possible or the design is limited by other more important factors than noise.

Passive noise reduction by absorbing and insulating acoustic elements is effective for high frequency noise but typically less effective for low frequency noise, as long waves require large elements. On the other hand, active noise control (ANC) is most effective for low frequency noise. Active attenuation is based on generating antisound with actuators, such that original noise is canceled. The antisound must have the same amplitude as the noise to be canceled, but the opposite phase so that destructive interference occurs. If the noise contains both high and low frequency components, the best noise attenuation is obtained by combining both active and passive methods together. It is challenging to estimate the effectiveness of local sound control in a complicated three-dimensional domain like in passenger cars and other vehicles. In the passenger car, low frequency noise sources are mainly due to structural vibration from engine and tires [18]. Especially structure-borne noises are low frequency, whereas airborne noises often have higher frequencies. Tires cause high frequency noise due to aerodynamic phenomena. The mechanical vibratory noise from tires is mainly below $1 \mathrm{kHz}$. The most important noise components originating in the passenger car engine are below $500 \mathrm{~Hz}$. The resonance of car cabin is also an important low frequency noise source. Accurate mathematical modeling of acoustics in such cabins is a formidable task [10].

As there are low-frequency noise sources, the local sound control can provide a significant noise reduction to the car cabin environment. More advanced methods designing and assessing such systems employ numerical simulation and optimization. The mathematical side of these problems have been considered in $[7,11,12]$, for example. Approaches using finite element modeling, are presented in articles [19, 5, 17]. In [19], resonance modes for mining vehicle are studied by modal coupling analysis and antinoise is optimized by using FEM model to obtain global noise control in the cabin. In [5], a local active noise control method based on the finite element method is described which minimizes noise locally in microphone locations. A method to determine the optimal locations for antinoise actuators is also presented. In [17], the locations of control sensors and actuators for global sound control are optimized based on finite element models for a complicated geometry. In [22], an optimal active noise control implementation based on quadratic programming and boundary element method (BEM) is presented.

Often it is necessary to control local sound in time varying domains. For example, parts of machinery move or like in this article, the driver of a car moves. It is convenient to use stochastic domains to model such changes in geometry instead of deterministic models. A vast amount of research has been performed on partial differential equations (PDEs) with stochastic coefficient, but PDEs in stochastic domains have had much less attention. In [21], a mapping from a random domain to a fixed domain is used to transform the problem to be one with stochastic coefficients. Fictitious domain approach is used in $[6,16]$ and an extended finite element method is employed in [15] to treat stochastic domains. For the local sound control problem considered here, a noise measure needs to 
be computed for a small subdomain. The expected noise can be conveniently computed in a stochastic domain by integrating numerically the product of the noise and the probability distribution function over possible domains. Thus, a functional of the solution of a stochastic PDE is computed directly without approximating the stochastic solution. This is a non-intrusive approach, that is, a solution method for non-stochastic problems like the one in $[1,2]$ can be employed without any modifications.

Here, a novel modeling method for the local control of sound by antinoise actuators is introduced in stochastic domains. It can be used to assess the possibilities of active noise control in enclosed acoustic spaces such as vehicle cabins. The method is based on using acoustic finite element modeling. The antinoise is optimized by minimizing the expected value of the noise computed using the finite element method. By including the stochasticity of the cavity domain in the model the optimal performance of a local sound control can be determined more accurately and reliably than with earlier methods. The numerical example, optimization of local sound control in a car cabin model, shows the efficiency of the presented method.

This article is organized as follows. In Section 2, a mathematical model of sound propagation, the Helmholtz partial differential equation, and a numerical method to solve it are briefly presented. In Section 3, the local noise control in a stochastic domain is formulated as a quadratic optimization problem. In Section 4, an example of local noise control in car driver's ears is described. In Section 5, the numerical results of ANC performance in three-dimensional car cabin problem are studied and analyzed. In Section 6, conclusions are given.

\section{Acoustic model}

The time harmonic sound propagation can be modeled by the Helmholtz equation

$$
-\boldsymbol{\nabla} \cdot \frac{1}{\rho} \nabla p-\frac{\omega^{2}}{c^{2} \rho} p=0 \quad \text { in } \Omega,
$$

where $\rho(\mathbf{x})$ is the density of the material at location $\mathbf{x}$, and $c(\mathbf{x})$ is the speed of sound in the material. The complex pressure $\hat{p}(\mathbf{x})$ defines the amplitude and phase of the pressure. The sound pressure at time $t$ is obtained as $e^{-i \omega t} p$, where $\omega$ is the angular frequency of sound and $i=\sqrt{-1}$. A sound source $f$ acting on a part $S$ of the boundary $\partial \Omega$ is modeled via a boundary condition. A partially absorbing wall material is described by the impedance boundary conditions

$$
\begin{array}{ll}
\frac{\partial p}{\partial \mathbf{n}}=\frac{i \eta \omega}{c} p+f & \text { on } S \\
\frac{\partial p}{\partial \mathbf{n}}=\frac{i \eta \omega}{c} p & \text { on } \partial \Omega \backslash S,
\end{array}
$$

where $\eta(\mathbf{x})$ is the absorption coefficient depending on the properties of the surface material. The value $\eta=1$ approximates a perfectly absorbing material and 
the value $\eta=0$ approximates a sound-hard material (the Neumann boundary condition).

An approximate solution for the partial differential equation (PDE) Eq. (1) can be obtained using a finite element method [20]. The finite element discretization transforms Eq. (1) into a system of linear equations $\mathbf{A x}=\mathbf{b}$, where the matrix $\mathbf{A}$ is generally symmetric, large, and sparse. Due to the large size and structure of $\mathbf{A}$, direct solution methods are computationally too expensive. Instead an iterative solution methods like GMRES needs to be used. Solving the system with a reasonable number of iterations is, however, challenging as the matrix $\mathbf{A}$ is badly conditioned and especially so when the calculation domain is large and the frequency is high. In the numerical example in Section 5, the solutions are computed after the systems are preconditioned by a damped Helmholtz preconditioner described in [1, 2].

\section{The noise control problem}

An acoustic model in an enclosed stochastic domain $\Omega(\mathbf{r})$ is considered, where $\mathbf{r}$ is a random vector that conforms to a known probability distribution $F(\mathbf{r})$. The pressure $p(\mathbf{x}, \mathbf{r}, \boldsymbol{\gamma})$ is the sum of the sound pressures caused by noise and $n$ antinoise sources

$$
p(\mathbf{x}, \mathbf{r}, \boldsymbol{\gamma})=p_{0}(\mathbf{x}, \mathbf{r})+\sum_{j=1}^{n} \gamma_{j} p_{j}(\mathbf{x}, \mathbf{r}),
$$

where the pressure amplitude $p_{0}$ is due to the noise source, $p_{j}$ is due to the $j$ th antinoise source, and $\gamma_{j}$ is a complex coefficient defining the amplitude and phase of the $j$ th antinoise source. The noise and antinoise sources are located on the boundaries of $\Omega$. The antinoise defined by the coefficients $\gamma_{j}$ is optimized so that the noise is minimized in a subdomain denoted by $\Xi(\mathbf{r}) \subset \Omega(\mathbf{r})$. For this, a noise measure is defined as

$$
\begin{aligned}
N(\mathbf{r}, \boldsymbol{\gamma}) & =\int_{\Xi(\mathbf{r})}|p(\mathbf{x}, \mathbf{r}, \boldsymbol{\gamma})|^{2} g(\mathbf{x}) d \mathbf{x} \\
& =\int_{\Xi(\mathbf{r})} p(\mathbf{x}, \mathbf{r}, \boldsymbol{\gamma}) \bar{p}(\mathbf{x}, \mathbf{r}, \boldsymbol{\gamma}) g(\mathbf{x}) d \mathbf{x},
\end{aligned}
$$

where $g(\mathbf{x})$ is a weighting function and $\bar{p}$ is the complex conjugate of $p$.

As the domain $\Omega$ is stochastic, the expected value of the noise measure is given by

$$
E(N(\mathbf{r}, \boldsymbol{\gamma}))=\int N(\mathbf{r}, \boldsymbol{\gamma}) F(\mathbf{r}) d \mathbf{r},
$$

where $F(\mathbf{r})$ is the probability distribution of $\mathbf{r}$. The objective function $J$ for optimization is chosen to be an approximation of the integral (5) and it is given 
by the numerical quadrature

$$
J(\boldsymbol{\gamma})=\sum_{j=1}^{m} w_{j} N\left(\mathbf{r}_{j}, \boldsymbol{\gamma}\right) F\left(\mathbf{r}_{j}\right),
$$

where the pairs $\left(\mathbf{r}_{j}, w_{j}\right)$ give the quadrature points and weights. The optimization problem is defined as

$$
\min _{\boldsymbol{\gamma} \in \boldsymbol{\Gamma}} J(\gamma)
$$

where $\boldsymbol{\Gamma}$ is the set of feasible controls. For example, in practical applications it is necessary to bound the amplitude of the antinoise sources.

In order to give the objective function in a compact form, the following notations are introduced:

$$
\begin{aligned}
\mathbf{p}(\mathbf{x}, \mathbf{r}) & =\left(p_{1}(\mathbf{x}, \mathbf{r}), p_{2}(\mathbf{x}, \mathbf{r}), \cdots, p_{n}(\mathbf{x}, \mathbf{r})\right)^{T}, \\
a & =\sum_{j=1}^{m} w_{j} F\left(\mathbf{r}_{j}\right) \int_{\Xi\left(\mathbf{r}_{j}\right)} p_{0}\left(\mathbf{x}, \mathbf{r}_{j}\right) \bar{p}_{0}\left(\mathbf{x}, \mathbf{r}_{j}\right) g(\mathbf{x}) d \mathbf{x}, \\
\mathbf{b} & =\sum_{j=1}^{m} w_{j} F\left(\mathbf{r}_{j}\right) \int_{\Xi\left(\mathbf{r}_{j}\right)} p_{0}\left(\mathbf{x}, \mathbf{r}_{j}\right) \overline{\mathbf{p}}\left(\mathbf{x}, \mathbf{r}_{j}\right) g(\mathbf{x}) d \mathbf{x}, \quad \text { and } \\
\mathbf{A} & =\sum_{j=1}^{m} w_{j} F\left(\mathbf{r}_{j}\right) \int_{\Xi\left(\mathbf{r}_{j}\right)} \mathbf{p}\left(\mathbf{x}, \mathbf{r}_{j}\right) \mathbf{p}^{H}\left(\mathbf{x}, \mathbf{r}_{j}\right) g(\mathbf{x}) d \mathbf{x},
\end{aligned}
$$

where $\overline{\mathbf{p}}$ is the elementwise complex conjugate of the vector $\mathbf{p}$, and the superscripts ${ }^{T}$ and ${ }^{H}$ denote the transpose and the Hermitian conjugate, respectively. By expanding terms and by using the notations in Eq. (8), the objective function in Eq. (6) can be expressed in a compact form

$$
J(\gamma)=\boldsymbol{\gamma}^{H} \mathbf{A} \boldsymbol{\gamma}+\boldsymbol{\gamma}^{H} \mathbf{b}+\mathbf{b}^{H} \boldsymbol{\gamma}+a .
$$

In the case that there are no constraints, that is, $\Gamma=\mathbb{C}^{n}$ in (7), the optimal complex coefficients $\gamma_{i}$ that give phases and amplitudes for antinoise actuators, are now given by the optimality condition $\nabla_{\gamma} J=\mathbf{0}$. This leads to a system of linear equations $\mathbf{A} \boldsymbol{\gamma}=-\mathbf{b}$, which has the solution

$$
\gamma=-\mathbf{A}^{-1} \mathbf{b}
$$

The case with constrains can be transformed to a real-valued optimization problem for a vector of length $2 n$ consisting the real and imaginary parts of $\gamma_{j} \mathrm{~s}$. The objective function has a quadratic form with a $2 n \times 2 n$ symmetric and positive definite matrix. There are several efficient methods available for such optimization problems (see [14]), and they could be applied for this problem. For technical simplicity, however, we restrict this work to the case with no constraints, which leads to the solution of linear systems of the form (10). 


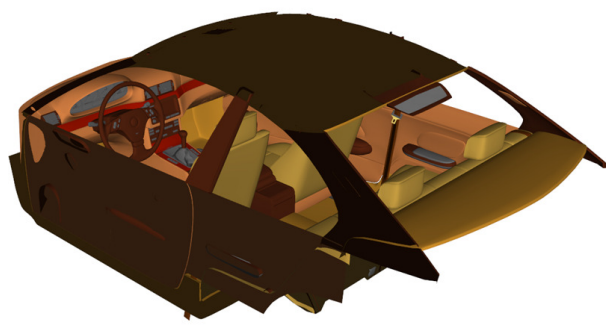

Figure 1: A three-dimensional model of BMW 330i car interior.

\section{Sound control in a car interior}

As an example of application of the numerical method, noise control in BMW 330i car interior is studied, see Fig. 1. The interior of the car excluding the driver is the domain $\Omega(\mathbf{r})$. The objective of the noise control is to minimize noise in driver's ears. Thus, $\Xi$ is defined as a set

$$
\Xi(\mathbf{r})=\left\{\mathbf{e}_{l}, \mathbf{e}_{r}\right\} \subset \Omega(\mathbf{r}),
$$

where $\mathbf{e}_{l}(\mathbf{r})$ and $\mathbf{e}_{r}(\mathbf{r})$ are the co-ordinates of the left and right ear, respectively. The noise measure in Eq. (4) has now the expression

$$
N(\mathbf{r}, \boldsymbol{\gamma})=\left|p\left(\mathbf{e}_{l}, \mathbf{r}, \boldsymbol{\gamma}\right)\right|^{2}+\left|p\left(\mathbf{e}_{r}, \mathbf{r}, \boldsymbol{\gamma}\right)\right|^{2} .
$$

It is assumed that there is only the driver and no other passengers or significant objects in the car that would influence the sound propagation. Driver's variable properties like shape and posture have an impact on reflections and propagation of sound, so they must be taken into account. Especially the posture and position of head affect the sound heard by ears. As the posture varies to some extent, it is better to minimize the expected value of the sound level in ears. This leads to a stochastic domain in the computation.

The driver is modeled by using the freely available Animorph library, that is based on ideas and algorithms presented in [3]. With Animorph, it is possible to model driver's geometry with a rich set of parameters changing the posture and shape. Three parameters are considered here: $r_{1}$ is driver's sideways bending angle, $r_{2}$ is forward bending angle, and $r_{3}$ is head rotation angle to left/right. These parameters are illustrated in Fig. 2.

Now the random variable $\mathbf{r}=\left(r_{1}, r_{2}, r_{3}\right)^{T}$ determines the posture of the driver, where the value of each parameter is limited by condition $L_{i}<r_{i}<H_{i}$, 

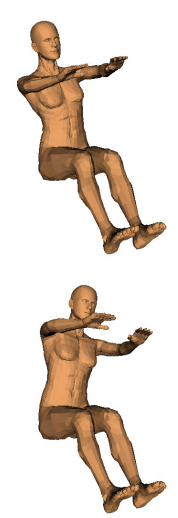

(a) $r_{1}$
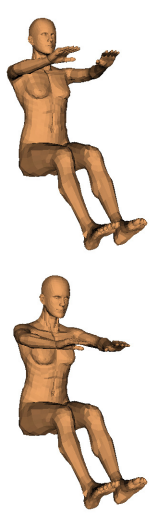

(b) $r_{2}$
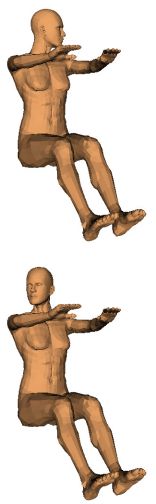

(c) $r_{3}$

Figure 2: Driver's posture parameters: (a) $r_{1}$ is driver's sideways bending angle, (b) $r_{2}$ is the forward bending angle, (c) $r_{3}$ is head rotation angle to left/right. Upper figures correspond to the lowest value of the parameter and lower figures correspond to the highest value of the parameter.

$i \in\{1,2,3\}$. The expected value in Eq. (5) reads now

$$
E(N(\mathbf{r}, \gamma))=\int_{r_{1}=L_{1}}^{H_{1}} \int_{r_{2}=L_{2}}^{H_{2}} \int_{r_{3}=L_{3}}^{H_{3}} N(\mathbf{r}, \gamma) F(\mathbf{r}) d r_{3} d r_{2} d r_{1} .
$$

The probability distribution function $F$ is given by a piecewise trilinear function defined by the nodal values on the lattice $\left\{L_{1}, C_{1}, H_{1}\right\} \times\left\{L_{2}, C_{2}, H_{2}\right\} \times$ $\left\{L_{3}, C_{3}, H_{3}\right\}$ and elsewhere by trilinear interpolation. The integral in Eq. (12) is estimated by the three-dimensional generalization of the trapezoidal quadrature rule. The numerical integration of expression in Eq. (12) gives the objective function

$$
J(\boldsymbol{\gamma})=E(N(\mathbf{r}, \boldsymbol{\gamma}))=\sum_{i=1}^{m} w_{i} N\left(\mathbf{r}_{j}, \gamma\right) F\left(\mathbf{r}_{j}\right)
$$

where $w_{i}$ is a weight coefficient from the trapezoidal rule for the integral of the probability distribution function $F$ and $\mathbf{r}_{i}$ is the co-ordinate triplet of the $i$ th quadrature point.

To evaluate the objective function in Eq. (13), the pressure amplitude caused by each noise and antinoise source is needed in ears for each driver sample $\mathbf{r}_{i}$. The acoustic reciprocity principle allows here a significant computational saving. The principle says that the observation stays the same when the locations of sound source and observer are exchanged. For more details about the principle and its applications, see $[9,8]$. This is employed in the following way. First, a finite element model which has a point noise source at the ear $\mathbf{e}_{i}$ is set up. Then, the pressure amplitude is studied in a noise or antinoise surface $S$. By the 
reciprocity the following holds: the sound emitted by the source $S$ measured at the ear $\mathbf{e}_{i}$ has the same pressure amplitude as the sound emitted from the ear $\mathbf{e}_{i}$ measured over the surface $S$. Thus, the sound pressure amplitude caused by many different sound sources can be resolved by just performing one simulation for each combination of sampled driver's posture, sampled frequency, and ear. The pressure amplitude heard at the ear $\mathbf{e}_{i}$ is given by the integral

$$
p_{S}\left(\mathbf{e}_{i}\right)=\int_{S} p_{\mathbf{e}_{i}}(\mathbf{x}) f(\mathbf{x}) d \mathbf{x},
$$

where $p_{S}\left(\mathbf{e}_{i}\right)$ is the sound pressure propagated from the surface $S$ that is heard at the ear $\mathbf{e}_{i}, f(\mathbf{x})$ is the force term for the sound source on $S$, and $p_{\mathbf{e}_{i}}(\mathbf{x})$ is the sound pressure propagated from the ear $\mathbf{e}_{i}$ at the point $\mathbf{x}$ on the surface $S$. With a point antinoise source $S$, the integral in Eq. (14) is replaced by the point value at $\mathrm{x}=S$.
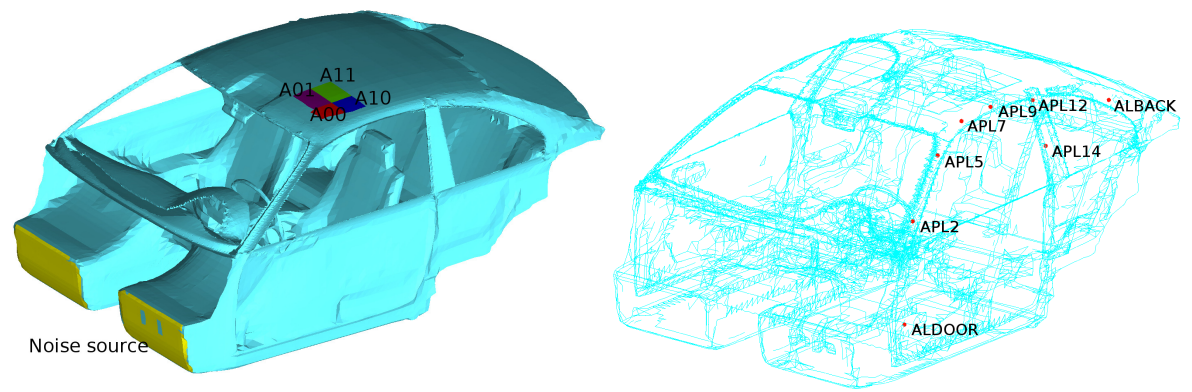

Figure 3: In the left figure, the noise source and planar antinoise sources are marked and labeled. In the right figure, there are point antinoise sources; only left side actuators are marked and labeled. The corresponding actuators on the right side are defined symmetrically on the right side of the cabin.

\section{Numerical experiments}

\subsection{Definition of the model}

In the numerical example model, the stochasticity of the domain $\Omega(\mathbf{r})$ implies that the Helmholtz equation Eq. (1) is solved many times with the driver in different postures. Table 1 lists the sampled values for the parameters $r_{1}, r_{2}$, and $r_{3}$. The center of the probability function $F$ is at $\mathbf{C}=(0,0,0)$ and the corners are at $\mathbf{L}=(-25,-7.5,-62.5)$ and $\mathbf{H}=(25,17.5,62.5)$. On the boundaries of the rectangular prism $\left[L_{1}, H_{1}\right] \times\left[L_{2}, H_{2}\right] \times\left[L_{3}, H_{3}\right]$, the probability function is set to zero, $F(\mathbf{r})=0$. 
Table 1: The parameter values for driver's stochastic variables $r_{i}$ are given on the second column. The third column gives the identification of the parameter used in Animorph library.

\begin{tabular}{l|c|c} 
Parameter & Values $\left[{ }^{\circ}\right]$ & Animorph parameter \\
\hline$r_{1}$, sideways bending & $-20,-10,0,10,20$ & $360 \_$torso/ROT1 \\
$r_{2}$, forward bending & $-5,0,5,10,15$ & $360 \_$torso/ROT2 \\
$r_{3}$, head rotation & $-50,-25,0,25,50$ & $300 \_$head/ROT2
\end{tabular}

To solve the Helmholtz equation in Eq. (1) with the finite element method, a collection of meshes consisting of linear tetrahedra and triangles were generated with Ansys ICEM CFD. Each mesh corresponds to different driver posture and they were generated so that there are at least 10 nodes per wavelength at $f=1000 \mathrm{~Hz}$. The total number of meshes is $5^{3}=125$ which is the number of parameter combinations $\left(r_{1}, r_{2}, r_{3}\right)$.

The noise and antinoise sources are presented in Fig. 3. The noise source is modeled by a uniformly vibrating surface behind the leg room, which is a simplification of the real noise source. The antinoise sources are labeled as follows. Antinoise panels on the roof are labeled as Axx, where $\mathrm{xx}$ is 00, 01, 10, and 11. Point actuators are labeled as AxDOOR, AxBACK, APx2, APx5, APx7, APx9, APx12, APx14, where $\mathrm{x}$ is here L for the left side sources and $\mathrm{R}$ is for the right side sources. Actuators AxDOOR are located on front doors and actuators APxxx are located on front side window frames, see Fig. 3. On inner surfaces, the absorbing boundary condition in Eq. (2) is posed with the absorbency coefficient $\eta=0.2$.

The study was done in the frequency range $50-1000 \mathrm{~Hz}$ with $25 \mathrm{~Hz}$ steps. This means that 39 frequencies are sampled. By employing the reciprocity principle a sound source is placed in an ear. The acoustic model is solved for all 125 sampled driver's postures for both ears. Thus, discrete Helmholtz equations are solved $125 \times 39 \times 2=9750$ times for the optimal antinoise control.

\subsection{Actuator quality evaluation}

It is possible to enhance the noise control by choosing good locations for antinoise actuators and by increasing their number. However, increasing the number of actuators also increases the costs and complexity of the noise control system. Thus, it is worthwhile to remove the actuators that have only minor contribution to the desired sound control. The graphs in Figs $4-6$ study the quality of noise control and evaluate how each actuator contribute to the noise control quality. 

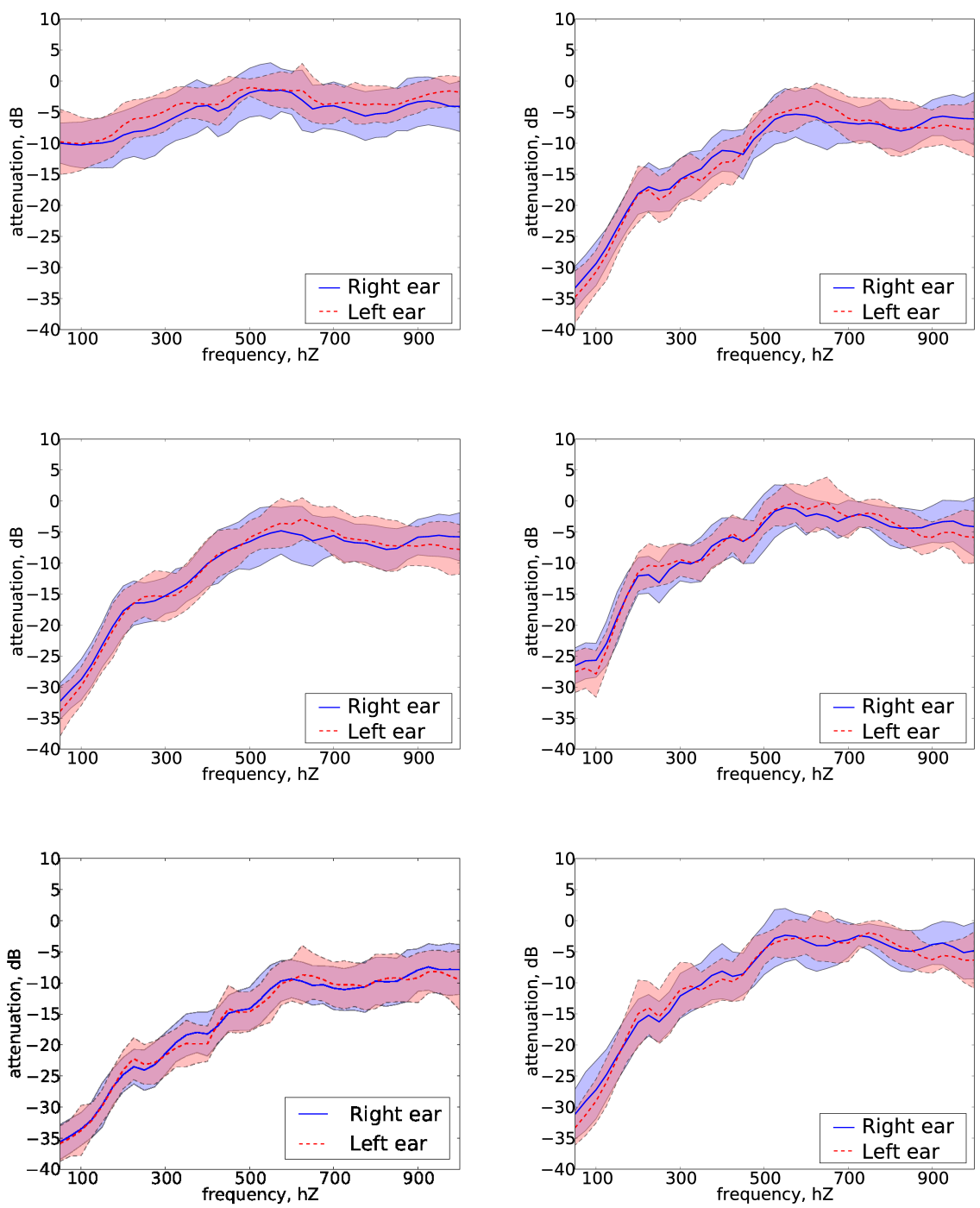

Figure 4: The expected value of attenuation in left and right ear with standard deviation $\sigma$ (shaded region). The antinoise actuators are used as follows: (a) Axx, (b) AxDOOR, AxBACK, Axx, (c) AxDOOR, Axx, (d) AxDOOR, (e) AxDOOR, APx2, APx5, APx7, APx9, APx12, APx14, (f) AxDOOR, AxBACK.

In Fig. 4, the expected value of the noise attenuation and its weighted standard deviation have been plotted at each driver's ear with different actuator combinations. By using two door loudspeakers (AxDOOR) as antinoise actuators, a satisfactory noise control is obtained within the engine noise frequency range, below $500 \mathrm{~Hz}$, as Fig. $4 \mathrm{~d}$ shows. By this choice, however, the noise 
reduction result is not good at higher frequencies, although the expected value of the attenuation stays negative, i.e. noise is reduced. In Fig. 4 e, 12 additional point antinoise actuators have been placed on side window frames. By these additional actuators, a good attenuation of ca. $10 \mathrm{~dB}$ is obtained over the whole studied frequency range.

In Figs 4 a and c, it can be seen that by using only planar actuators on the roof $(A x x)$, the attenuation at low frequencies is not good, but at higher frequencies $(700-900 \mathrm{~Hz})$ it is reasonable. When comparing Figs $4 \mathrm{c}$ and d, it is clear that the planar roof actuator $(\mathrm{Axx})$ together with the side door actuators (AxDOOR) is significantly better than the side door actuators (AxDOOR) alone. The attenuation profile is more flat, and even at high frequencies $(600-1000 \mathrm{~Hz})$ more than $5 \mathrm{~dB}$ expected attenuation is obtained.

If the antinoise itself is very loud, it may cause high sound pressure levels in some parts of the car cabin. By good placement, the amplitude of each actuator can be kept comfortable. In Fig. 5, the amplitude of each antinoise actuator is plotted to evaluate the actuator selections. Fig. 5 a shows that the amplitude of planar roof loudspeakers $(\mathrm{Axx})$ is over $10 \mathrm{~dB}$ louder than the amplitude of front door loudspeakers (AxDOOR), especially at high frequencies. In Fig. 5 $\mathrm{b}$, it is seen that the amplitude of back window loudspeakers (AxBACK) is significantly lower than the amplitude of front door loudspeakers (AxDOOR). However, when comparing Figs $4 \mathrm{~d}$ and $\mathrm{f}$, it is seen that the contribution of back window loudspeakers (AxBACK) is insignificant for the noise control.
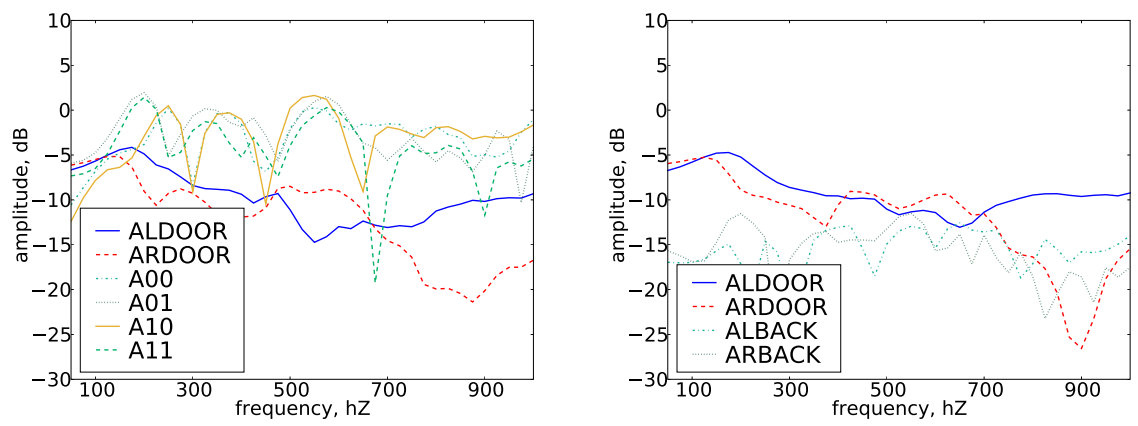

Figure 5: The amplitude of antinoise actuators is plotted in $\mathrm{dB}$ with the unit value $\left|\gamma_{i}\right|=1$ being $0 \mathrm{~dB}$. The antinoise actuators are used as follows: (a) AxDOOR, Axx, (b) AxDOOR, AxBACK.

In Fig. 6, the contribution of each actuator to the noise control is plotted in the following way. The noise levels are compared in both ears when the chosen actuator is enabled and when it is disabled, i.e. $\gamma_{i}=0$. For each examined frequency, the worst attenuation result is selected from left or right ear. From Fig. 6, the benefit of each actuator can be evaluated. As already has been suggested, it is seen from Fig. $6 \mathrm{~d}$ that the contribution of back window 
actuators (AxBACK) is less significant than of other actuators, and at high frequencies it is negligible.
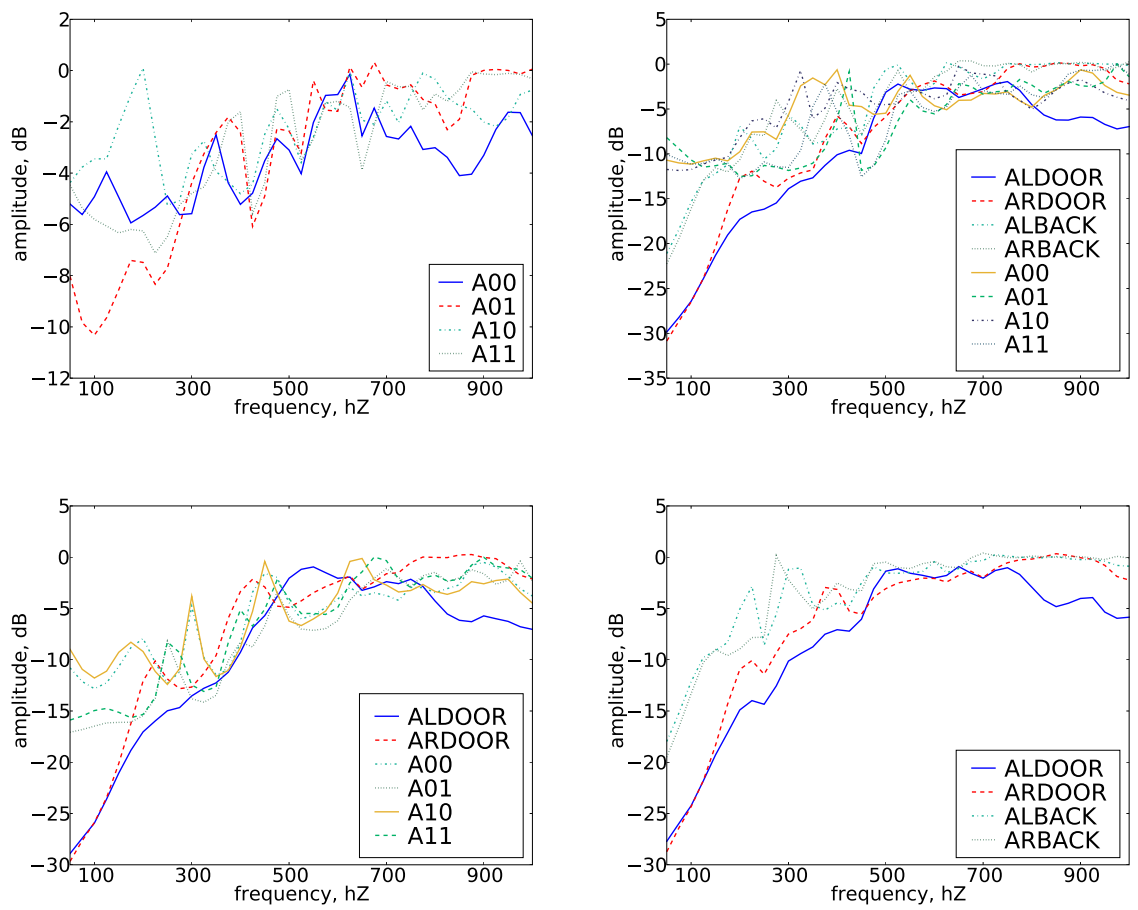

Figure 6: The contribution of each actuator to the noise control system. The noise level is compared between the case when the inspected actuator is enabled and when it is disabled, i.e. $\gamma_{i}=0$. For each examined frequency, the worst attenuation is selected from left or right ear and it is plotted in the graph in dB. The antinoise actuators are used as follows: (a) Axx, (b) AxDOOR, AxBACK, Axx, (c) AxDOOR, Axx, (d) AxDOOR, AxBACK.

\subsection{Attenuation plots}

In Fig. 7, there are example plots of the attenuation when the driver is at different postures. The two front door loudspeakers (AxDOOR), the back window $(\mathrm{AxBACK})$ and the planar roof $(\mathrm{Axx})$ loudspeakers are used as the antinoise actuators. When the frequency is less than $400 \mathrm{~Hz}$, there is more than $10 \mathrm{~dB}$ attenuation in almost every posture. At frequencies higher than $400 \mathrm{~Hz}$, there is mostly significant, over $5 \mathrm{~dB}$ attenuation, but there are also occasional postures that lead to noise amplification, i.e. additive interference of sounds. However, strong noise peaks are unlikely and on average the noise is reduced significantly. 

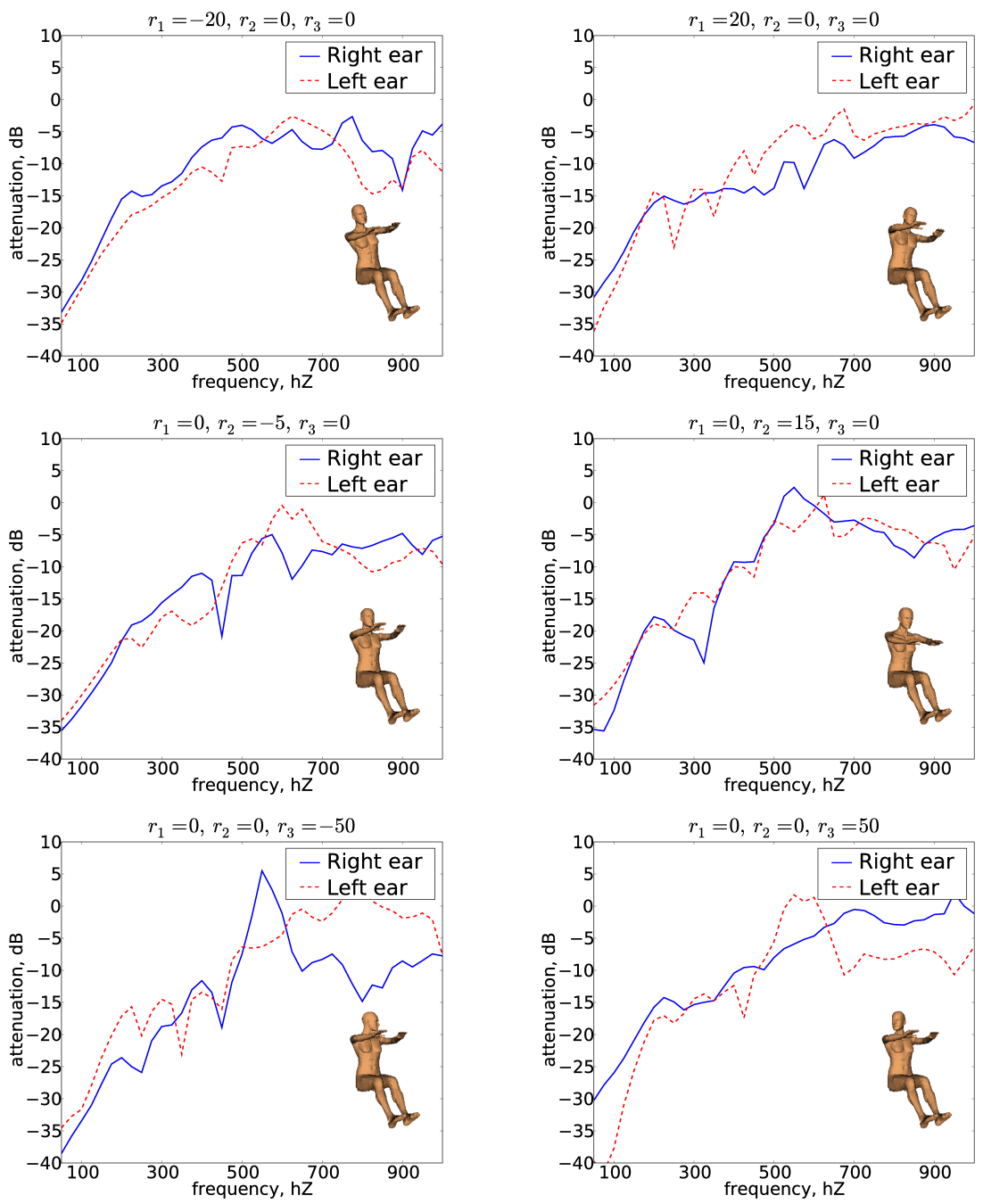

Figure 7: Examples of attenuation graphs at different postures as a function of frequency. The front door (AxDOOR), the back window (AxBACK), and the planar roof $(\mathrm{Axx})$ antinoise actuators are used in the noise control.

Fig. 8 demonstrates the effect of active noise control with two actuators (AxDOOR) at single frequency $f=300 \mathrm{~Hz}$. It is seen that the method reduces noise effectively near the ears and also in a wider region around the ears. At higher frequencies, the silent area is smaller and the noise is increased in other parts of the car. 


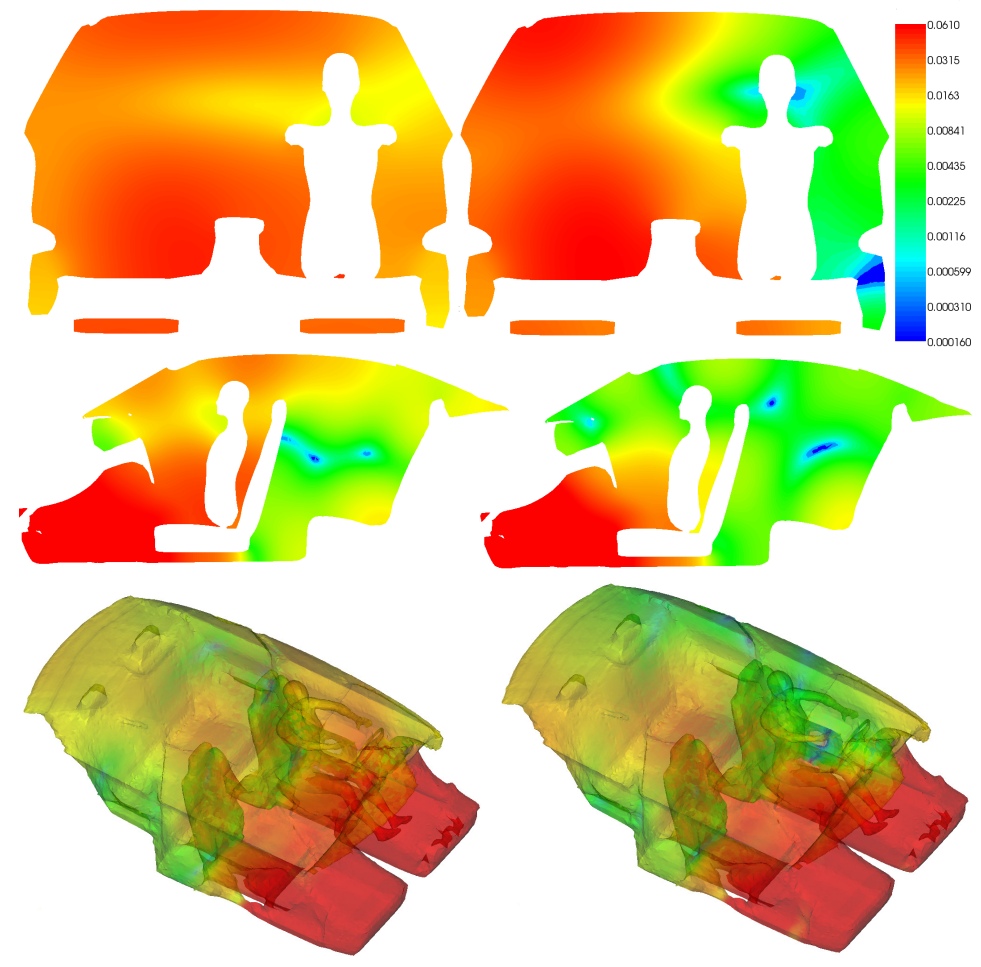

Figure 8: The noise control at frequency $f=300 \mathrm{~Hz}$ for the basic driver's parameters $r_{1}=r_{2}=r_{3}=0$. The modulus of pressure amplitude $|p|$ is plotted on the logarithmic color scale. On left plots, the acoustic field is depicted without noise control. On right plots, the noise control is enabled. The attenuation at both ears in this case is ca. $-30 \mathrm{~dB}$. The front door (AxDOOR) loudspeakers are used in the noise control.

\section{Conclusions}

A novel method is introduced to assess the effectiveness of the optimal antinoise for local sound control in a stochastic domain. The acoustic modeling is performed in the frequency domain using a sequence of finite element discretizations of Helmholtz equations. The optimization of antinoise is performed by minimizing the expected value of the noise at each frequency. This leads to a robust and accurate noise control in varying domains.

The sound control in a car interior with a driver in varying postures is considered as an example and numerical results are presented. A good attenuation noise is obtained for probable postures at lower frequencies, say, below $300 \mathrm{~Hz}$. At higher frequencies, the noise reduction can be improved by increasing the number of actuators. 


\section{References}

[1] T. Airaksinen, E. Heikkola, A. Pennanen, and J. ToivaNEN, An algebraic multigrid based shifted-Laplacian preconditioner for the Helmholtz equation, J. Comput. Phys., 226 (2007), pp. 1196-1210.

[2] T. Airaksinen, A. Pennanen, and J. Toivanen, A damping preconditioner for time-harmonic wave equations in fluid and elastic material, J. Comput. Phys., 228 (2009), pp. 1466-1479.

[3] M. Bastioni, S. Re, And S. MisRa, Ideas and methods for modeling $3 D$ human figures, in Proceedings of the 1st Bangalore Annual Compute Conference, ACM, 2008.

[4] L. L. Beranek and I. L. Ver, Noise and Vibration Control Engineering. Principles and Applications, John Wiley, New York, 1992.

[5] A. Bermúdez, P. Gamallo, and R. Rodríguez, Finite element methods in local active control of sound, SIAM J. Control Optim., 43 (2004), pp. 437-465.

[6] C. Canuto and T. Kozubek, A fictitious domain approach to the numerical solution of PDEs in stochastic domains, Numer. Math., 107 (2007), pp. 257-293.

[7] F. Fahroo And M. A. Demetriou, Optimal actuator/sensor location for active noise regulator and tracking control problems, J. Comput. Appl. Math., 114 (2000), pp. 137-158.

[8] F. Fahy and P. Gardonio, Sound and Structural Vibration, Academic Press, 2006.

[9] F. J. FAHY, Some applications of the reciprocity principle in experimental vibroacoustics, Acoust. Phys., 49 (2003), pp. 217-229.

[10] F. Ihlenburg, Sound in vibrating cabins: Physical effects, mathematical formulation, computational simulation with fem, in Computational Aspects of Structural Acoustics and Vibration, R. Ohayon and G. Sandberg, eds., Springer, 2009, pp. 103-170.

[11] J. LonČARIĆ And S. V. Tsynkov, Optimization of power in the problems of active control of sound, Math. Comput. Simulation, 65 (2004), pp. 323-335.

[12] — Quadratic optimization in the problems of active control of sound, Appl. Numer. Math., 52 (2005), pp. 381-400.

[13] P. A. Nelson and S. J. Elliot, Active Control of Sound, Academic Press, London, 1999. 
[14] J. Nocedal AND S. J. WRIGHT, Numerical optimization, Springer Series in Operations Research and Financial Engineering, Springer, New York, second ed., 2006.

[15] A. Nouy, A. Clément, F. Schoefs, And N. Moës, An extended stochastic finite element method for solving stochastic partial differential equations on random domains, Comput. Methods Appl. Mech. Engrg., 197 (2008), pp. 4663-4682.

[16] L. Parussini and V. Pediroda, Investigation of multi geometric uncertainties by different polynomial chaos methodologies using a fictitious domain solver, CMES Comput. Model. Eng. Sci., 23 (2008), pp. 29-51.

[17] C. G. Provatidis, S. T. Mouzakitus, and G. N. CharalamPOLOUlos, Simulation of active noise control in enclosures using direct sound field prediction, J. Comput. Acoustics, 17 (2009), pp. 83-107.

[18] P. SHORTER, Recent advances in automotive interior noise prediction. SAE BRASIL Noise and Vibration Conference, 2008.

[19] D. A. Stanef, C. H. Hansen, and R. C. Morgans, Active control analysis of mining vehicle cabin noise using finite element modelling, J. Sound Vib., 277 (2004), pp. 277-297.

[20] L. L. Thompson, A review of finite-element methods for timeharmonic acoustics, J. Acoust. Soc. Am., 119 (2006), pp. 13151330 .

[21] D. Xiu and D. M. Tartakovsky, Numerical methods for differential equations in random domains, SIAM J. Sci. Comput., 28 (2006), pp. 1167-1185.

[22] T. C. Yang And C. H. Tseng, Effective optimization-based approach for designing active noise control systems in enclosures, Finite Elem. Anal. Des., 15 (1994), pp. 303-316. 